\title{
ÉCRITURE SAVANTE ET RELATION AU VOYAGE
}

\author{
DANIELE LAPLACE-TREYTURE ${ }^{1}$
}

Résumé: Cet article a pour objet la relation entre l'écriture savante (ici l'approche géographique des lieux) et l'expérience de l'altérité liée au déplacement. A partir d'une prise en compte de la dimension énonciative du discours, à travers notamment un rapport de l'énonciateur au langage, on met en évidence diverses formes d'altérité présentes dans le texte savant. L'ouverture du discours vers l'altérité est ensuite envisagée comme offrant la possibilité du discours géographique d'intégrer différentes appréhensions des lieux, et par là d'en présenter une image complexe.

Mots-clés: Discours géographique, genre régional, énonciation, altérité, lieu, Vidal de La Blache.

\begin{abstract}
SCHOLARLY WRITING AND TRAVELS- This paper will examine the relationship between the geographic discourse on place and the experience of otherness, or alterity. Through the enunciation dimension of discourse, one can find signs in texts that indicate the degree to which the discourse is open to otherness. The openning of discourse to alterity offers to geographic discourse the possibility of integrating different meanings to place and therefore presenting more complex representations.
\end{abstract}

Key-words: Geographic discourse, regional geography, enunciation, otherness, place, Vidal de La Blache

Resumo: DISCURSO ACADÉMICO RELATIVAMENTE ÀS VIAGENS - Este texto examina a relação entre o discurso geográfico sobre os lugares e a experiência do outro, ou alteridade. A partir da tomada de consciência da dimensão enunciativa do discurso, procuram-se indícios nos textos que mostrem de que forma o discurso se dirige ao outro. A abertura do discurso à alteridade oferece a possibilidade de integrar diferentes significados aos lugares e, assim, de apresentar representações mais complexas.

Palavras-chave: Discurso geográfico, geografia regional, enunciação, alteridade, lugar, Vidal de La Blache.

1 Maître de Conférences à l'Université de Pau et des Pays de l'Adour.

Laboratoire Société Environnement Territoire. C.N.R.S. U.M.R.-5603. Domaine Universitaire, 64000 Pau, France.

Tel.: 559.92.31.20; Fax: 559.80.83.39; E-mail:daniele.laplace@univ-pau.fr 
Il existe un type d'écrits savants appartenant au genre régional - genre géographique ancien - qui offre un bel exemple des rapports ambivalents que l'écriture savante noue avec l'expérience liée au déplacement, qu'on la nomme "voyage scientifique" ou "terrain". En effet, dans ces écrits, l'expérience liée au déplacement, et à travers celui-ci, l'expérience même de l'altérité, occupe une place complexe, sinon ambiguë. Cette dernière fonde le discours, ou tout au moins en est souvent le point de départ; pourtant, dans le texte final, elle se trouve partiellement, voire parfois complètement, occultée. Plus exactement, l'altérité et l'expérience de l'altérité sont diversement et savamment mises à distance.

Au-delà de la diversité des épistémologies et des genres en géographie, peut-on concevoir un discours géographique ayant l'homme ou les sociétés humaines pour "objet" qui serait en même temps dépourvu de toute trace d'un rapport à autrui, et plus largement à l'altérité? Ne peut-on pas alors imaginer que cette altérité, passée au filtre de catégories et de dispositifs d'enquête scientifiques toujours trop réducteurs au regard de la pluralité du monde, se trouve en partie reconstruite (voire transfigurée) dans et par le texte final? Si tel était le cas, cela ramènerait l'attention sur le passage problématique et stratégique à l'écriture.

Dans le texte qui suit, je propose une réflexion sur les modalités et le sens que pourrait recouvrir l'inscription de l'Autre (non seulement autrui mais aussi d'autres formes d'altérité moins évidentes) dans le discours géographique savant. Dans un premier temps, je précise le contenu d'un questionnement sur l'Autre et sur l'altérité en général dans le cadre d'une analyse du discours géographique. Le deuxième temps montre comment la question d'une présence/absence de l'Autre dans le discours peut être posée par le biais d'une problématique de l'énonciation. À travers le jeu de l'inscription du Sujet et de ce qui lui est extérieur, étranger (c'est-à-dire autre), il est possible d'apprécier un degré d'ouverture du discours vers diverses formes d'altérité. En dépit de modes énonciatifs oscillant entre dénégation de soi et oubli de l'Autre, le discours savant, en l'occurrence géographique, admet parfois une ouverture vers l'altérité, et ce, sous des formes (ou des modalités) textuelles tout à la fois diverses, inattendues et subtiles. Dans un troisième temps, je replace l'enjeu d'une prise en compte de l'altérité dans la perspective de l'approche géographique des lieux au sens large ${ }^{2}$. Ce dernier point s'achève par quelques remarques sur l'écriture du Tableau de la Géographie de la France de Paul Vidal de La Blache publié en 1903.

\section{LE CONTENU D'UNE PROBLÉMATIQUE DE L'ALTÉRITÉ EN GÉOGRAPHIE}

Parmi les expériences liées au déplacement, il y a celle de l'altérité que ce dernier permet de faire, appréhension de la diversité des individus, des choses et des lieux, dans

2 Dans le cadre de cet article, "approche géographique des lieux" et "genre régional" sont pour moi synonymes. A la suite de V. Berdoulay, je définis ce dernier comme "visant l'intégration des données éparses ou, dit autrement, de la totalité, ou encore des interdépendances qui régissent la vie sociale à une échelle donnée" (BERDOULAY, 1988, p. 17). 
ce qu'ils ont de différent ou de nouveau, peut-être même d'inédit, et qui en fait des "objets" difficiles à penser et à communiquer. Aux difficultés d'ordre conceptuel appréhender par exemple la spécificité d'un rapport homme-environnement à travers des catégories d'analyse adéquates - vient s'ajouter celle de donner forme à un discours, qui renvoie plus directement à une prise de conscience des enjeux liés à l'écriture. Sans aller jusqu'à invoquer cette part "irréductible" de l'Autre qui ne peut manquer d'interroger profondément celui qui se déplace à la rencontre des autres, l'idée d'altérité inclut aussi, et peut-être surtout, celle d'un discours scientifique qui se construit en s'exposant, ou mieux, en s'articulant, à d'autres discours, à d'autres modes de connaissance de la réalité qui ne sont pas uniquement savants. Dès lors que l'on s'intéresse à l'écriture savante, il me parait important de bien identifier ces deux figures de l'altérité que sont l'Autre-habitant et l'Autre-lecteur, tous deux n'appartenant pas nécessairement au même lieu et/ou à la même culture et ne disposant pas non plus du même univers de références. Le géographe dit en effet quelque chose à propos de quelqu'un à quelqu'un d'autre; il est un passeur entre des mondes qu'il cherche à rendre commensurables, le monde de l'Autre-habitant et le monde du lecteur.

La relation de voyage est, il est vrai, le genre de prédilection des expériences liées au déplacement. A travers le récit d'un rapport sensible au monde, la relation de voyage constitue une forme particulière d'appréhension des lieux. Sa force réside, entre autres, dans le fait de savoir capter l'attention du lecteur, mais aussi de lui donner un rôle essentiel, actif, dans la construction du sens en l'obligeant à mobiliser son propre univers de perception et de connaissances pour le confronter à celui du texte. D'où un sens qui n'est pas d'emblée donné mais qui naît de la rencontre effective, dans la lecture, d'un univers d'écriture et d'horizons de lecture différents. Par là aussi, la relation de voyage peut créer les conditions d'accés à un savoir non-littéraire des lieux, et dans ce sens, recèle probablement de précieux leviers susceptibles d'enclencher le processus d'acquisition de connaissances plus spécifiquement savantes. En exposant le sujet à de multiples péripéties l'amenant tout autant à découvrir l'Autre qu'à faire retour sur soi et se retrouver soi-même, ce genre littéraire se prête tout particulièrement à l'étude des rapports de l'identité à l'altérité.

Mais les expériences liées au déplacement suscitent d'autres écritures, notamment celle savante à laquelle je m'intéresse ici. Il s'agit alors d'écrits ni semi-littéraires ni semi-scientifiques, mais qui appartiennent au discours savant et se conforment à des règles discursives propres à ce dernier, c'est-à-dire qu'ils répondent à d'autres habitudes de lecture et d'écriture. En tant que type de discours - différent par exemple du discours littéraire - le discours savant se focalise moins sur le sujet que sur l'objet. Par ailleurs, les textes manifestent un effacement de l'auteur, sont plutôt dépersonnalisés, indépendants du contexte, tendent vers une certaine transparence du langage et confèrent au propos un sens univoque, des caractéristiques censées servir la fonction de connaissance du discours scientifique. C'est là un mode d'énonciation caractéristique des discours savants dans leur grande majorité.

A propos de l'expérience de terrain des ethnologues, et plus largement du travail 
d'enquête et d'observation, de nombreux auteurs se sont attachés à montrer comment cette "pratique du terrain" était intégrée et reconstruite par le texte qui met en avant, sinon en scène, la figure de l'homme de terrain, garante de l'objectivité des "faits" observés et soumis à l'analyse (MONDADA, 1994). A travers cette figure de l'homme de terrain - cas de l'ethnologie - on mesure combien ce qui subsiste du voyage n'est plus tout à fait le sens de l'altérité dans ce qu'elle a de difficilement accessible, mais plutôt l'idée d'un milieu, d'un lieu (voire d'un groupe humain) déjà peu ou prou maîtrisés, à reconnaître plutôt qu'à connaître. La figure de l'homme de terrain vient donc se substituer à l'expérience même de l'altérité peu compatible, quant à elle, avec un discours de connaissance, tout au moins tant qu'elle reste perçue comme quelque chose de flou, d'irréductible, et par essence de largement indicible.

Ce n'est pas au terrain, figure en quelque sorte appauvrie du voyage et de l'altérité, auquel je m'intéresse ici. Je souhaite plutôt revenir au voyage et à l'apprentissage de l'altérité qu'il suppose pour m'interroger sur la possibilité, tout comme sur l'importance, compte tenu ou en dépit des finalités mêmes du discours savant, d'en retrouver la trace dans le texte final. Quelles formes d'altérité (ou de rapport à l'Autre) le texte peut-il inscrire?

\section{LE DÉTOUR PAR L'ÉNONCIATION, RÉVÉLATRICE D'UNE OUVERTURE DU DISCOURS VERS L'ALTÉRITÉ}

Je propose en effet de saisir l'ouverture du discours vers l'altérité par le biais de son mode d'énonciation. L'idée est de situer une telle ouverture non pas sur un plan conceptuel mais plutôt énonciatif. Autrement dit, il s'agit d'être attentif aux marques textuelles de l'inscription du Sujet et de l'Autre. Pour Jacqueline Authier, dont j'ai suivi les propositions, étudier l'énonciation, cela consiste à rechercher les moyens qu'a l'énonciateur - ici le géographe-auteur - de marquer une place à l'Autre, et ainsi d'inscrire une certaine distance à l'altérité (AUTHIER-REVUZ, 1982 et 1984). En se focalisant plus particulièrement sur un rapport du sujet au langage, cet auteur relève diverses formes de représentation de l'altérité dans le texte. Ainsi, le texte peut désigner l'Autre à travers:

- l'italique, les guillemets, ou bien le discours indirect;

- un autre discours (technique par exemple);

- une autre langue, un autre registre discursif.

A cet égard, on peut mentionner le recours à des termes vernaculaires; la description des lieux, par le biais notamment de la toponymie, y est propice, introduisant par là de nouvelles manières langagières d'ouvrir un discours à l'altérité.

En outre, ce sens de l'Autre apparaît également à travers d'autres marques de langage qui mobilisent le lecteur de façon, me semble-t-il, originale et forte, nouant un véritable dialogue avec lui. En effet, la présence d'un mot "potentiel ou explicite dans les figures de la réserve, de l'hésitation et de la rectification", indique aussi un autre 
moyen par lequel ce sens de l'Autre se trouve objectivé et est susceptible de lecture. Cet effort de distanciation de l'auteur, non seulement à l'égard de son objet d'étude autrui, les sociétés humaines dans leur relation à l'environnement - mais aussi vis-à-vis de son propre discours (on parlerait aujourd'hui de réflexivité), appelle l'existence d'un lecteur capable, quant à lui, de déchiffrer les signes d'une lecture non pas hésitante mais interrogative du monde, et par là-même ouverte à l'altérité.

Au-delà de ces formes de présence de l'Autre assez circonscrites, il est aussi possible de montrer tout un jeu de distanciation à l'altérité passant par le biais de portions bien plus importantes de texte que représentent, particulièrement en géographie, les descriptions. Selon l'entrée choisie (esthétique, en termes d'espace vécu...), celles-ci véhiculent alors diverses appréhensions possibles d'un lieu.

\section{DES FORMES ÉNONCIATIVES CENTRÉES SUR L'AUTRE À LA COMPLEXITÉ DES LIEUX}

Relativement à l'approche des lieux, on peut penser qu'en réduisant les registres d'interprétation, en appliquant des procédures ou des dispositifs d'enquête plus ou moins simplificateurs et stéréotypants de par leur unicité, le discours savant instaure une "distance fixe à l'objet". Parallèlement, celui-ci se coupe de la diversité des expériences, pratiques et représentations des lieux, qui constituent autant de dimensions par lesquelles ceux-ci font sens pour le lecteur.

Certaines marques d'altérité présentes dans le texte, renvoyant soit à l'Autre-habitant soit à l'Autre-lecteur, peuvent indiquer une volonté d'intégrer une approche ou un point de vue non-scientifiques sur les lieux, certes pour s'en distinguer, mais aussi dans le but de rompre avec le caractère généralement monologique des discours géographiques savants classiques. Ceci permet de mettre en perspective des points de vue différents sur les lieux et de donner de ces derniers une représentation non pas équivoque mais riche et ouverte à l'altérité. Cependant, l'enjeu n'est pas seulement communicationnel - à travers une dimension pédagogique ou didactique de certains textes - mais aussi et plus fondamentalement épistémologique car le lieu apparaît à travers le jeu de regards croisés créant une distance non plus fixe mais mobile à l'objet.

Parmi les géographes qui s'intéressent à l'approche régionale, la nécessité de se situer par rapport à d'autres visions de l'espace est d'ailleurs une question qui a été débattue. Que l'on relise à ce propos certaines réflexions sur l'écriture régionale aujourd'hui ${ }^{3}$. Les contributions sont en effet jalonnées d'interrogations sur le regard ou le point de vue à adopter. Certains géographes se demandent par exemple s'il faut opposer une Afrique du chercheur-témoin et une Europe du chercheur-acteur, si l'on doit mêler le point de vue savant et le regard de l'habitant-observateur. Les questions portent sur les angles de vue à retenir et sur leur articulation possible. Cette interroga-

\footnotetext{
3 Il s'agit de débats organisés à l'occasion dela rédaction de la dernière Géographie Universelle sous la direction de Roger Brunet (1990), dont des comptes rendus ont été publiés notamment dans l'Espace Géographique et dans Géopoint.
} 
tion est liée à la crainte d'aboutir à une représentation des lieux qui n'aurait d'existence que dans le champ de la science et plus aucun sens au regard de l'univers de connaissance et de perception du lecteur. Ces mêmes questions paraissent sous-tendues par une conception d'un genre régional dont la source de légitimité (ou de sens) résiderait dans une communauté de lecteurs...et de lectures possibles. Certains lui assignent une fonction sociale importante dans la formation de l'individu.

Josefina Gómez Mendoza et Nicolas Ortega Cantero résument bien la difficulté de mêler harmonieusement les langages, voire les genres: "Poètes et narrateurs, chroniques de voyage et journalisme, tout peut aider à retrouver le style que réclame l'écriture sur la région, aussi loin des concepts trop abstraits de la théorie que de la mise en scène trop personnalisée et affective" (GOMEZ MENDOZA et ORTEGA CANTERO, 1989: 96). On peut également citer Giuseppe Dematteis qui, d'un côté, met en rapport certaines types d'attentes (être renseigné, avoir une connaissance ordonnée et systématique d'un espace telle qu'elle serait utile par exemple aux décideurs) et une écriture qui évoque pour lui la stratégie, et de l'autre, associe "le versant de la créativité" et la forme du récit, dont la finalité serait plutôt de produire un texte attrayant aux yeux des lecteurs. Mais, ajoute-t-il, "je ne sais pas comment on peut composer les deux" (DEMATTEIS, 1993: 269). Il reste cependant que l'approche régionale hésite à se placer en dehors du spectacle du monde et à privilégier, à l'exclusion de tout autre, un point de vue trop décentré et décontextualisé, c'est-à-dire, comme je l'ai déjà évoqué plus haut, coupé de la réalité mondaine des individus. Il apparaît en tout cas que le souci, au départ quelque peu secondaire, de satisfaire les attentes souvent mal définies du public revient comme condition de possibilité d'un discours qui existe d'abord par le fait qu'il s'adresse à l'Autre.

Cette capacité à multiplier les angles de vue et à adopter un regard mobile à l'égard de l'objet est un des aspects essentiels de l'approche régionale telle que Paul Vidal de La Blache la met en œuvre dans son Tableau de la Géographie de la France (VIDAL DE LA BLACHE, 1903). La transmission d'un savoir géographique savant sur la France est en effet ici de l'ordre d'une véritable initiation à un regard géographique, région après région, pays après pays, voire lieu après lieu. L'accès à la connaissance se joue dans l'entre-deux d'un voir-savoir, dont le médiateur premier est le regard. Chez Vidal, l'observation (l'action de voir et les descriptions qui en résultent) s'inscrit fondamentalement dans un rapport ouvert à autrui, à d'autres appréhensions des lieux; Vidal regarde à la fois "avec" et "comme" l'Autre. "Comme" l'Autre, le non-spécialiste, lorsqu'il fait appel à un regard rassembleur et évoque le paysage à travers un répertoire de formes connues et culturellement éprouvées. "Avec" l'Autre car il ne récuse pas cette approche sensible, mais sans originalité, des lieux. Le regard joue donc ici un rôle essentiel: il se débouble, se fractionne même en plusieurs façons de regarder, ellesmêmes à l'origine de différents points de vue et savoirs sur les lieux. Mais l'œil du géographe n'est pas omniscient, ce qui le rendrait peu crédible et totalement inhumain. Au contraire, il est partagé: l'Autre habite ce regard, l'interroge, le rend pluriel tout en ne mettant pas en péril l'énonciateur dans son identité discursive. En effet, à aucun moment le texte vidalien introduit une confusion des registres ou des genres: le propos 
est d'un bout à l'autre scientifique, mais, et c'est là sa modernité, largement ouvert à l'altérité.

\section{EN GUISE DE CONCLUSION}

A travers le premier terme - "écriture savante" - de l'intitulé de cette contribution, j'entendais attirer l'attention sur le temps de l'écriture, dans la mesure où cette dernière convoque d'autres formes d'altérité, d'autres expériences de la relation à l'Autre. Ainsi la dimension de l'énonciation rappelle-t-elle que l'ouverture vers l'altérité n'est point seulement une affaire de concept mais aussi d'écriture et (re)découvre-t-on le lecteur comme une figure à part entière de l'altérité. On pourrait avancer l'idée selon laquelle, dans le texte final, le silence imposé à l'Autre-habitant se trouve en quelque sorte contre-balancé par l'existence du "dialogue" que nouent, à un autre niveau, l'auteur et le lecteur. Quand bien même les genres savants n'ont pas vocation à rapporter une expérience sensible des lieux, un sens de l'Autre, un rapport du sujet à l'altérité peut très bien s'y manifester, d'abord comme questionnement des limites du discours de la science. La nouveauté est du côté d'un changement des codes d'écriture tout autant que du côté des habitudes de lecture des textes savants. Cela suppose que l'on s'interroge sur la créativité des formes discursives elles-mêmes.

En rappelant brièvement l'exemple du Tableau de la Géographie de la France, mon intention n'était pas d'identifier des solutions idéales pour régler la question de l'appréhension savante des lieux, mais plutôt d'évoquer une écriture vive qui suggère un usage plus souple du discours savant, appelle une lecture sans doute moins convenue, moins normative, de ce même discours, et renvoie en somme à un savoir savant qui se construit dans un rapport ni uniforme ni univoque à l'altérité.

On établit souvent une analogie entre le voyage et l'écriture. Au-delà de l'aspect assez convenu de cette dernière, Philippe Dubois rappelle que toute forme de voyage renvoie à une pratique d'écriture spécifique (DUBOIS, 1981). Inversement, on peut dire que l'écriture savante porte en elle une approche du voyage qui tend fortement à en réduire, à en modifier l'altérité. Dès lors, il me paraît légitime d'envisager l'existence d'autres espaces de représentation de l'altérité, d'autres lieux d'expression du Sujet et de l'Autre. L'espace ainsi libéré et parcouru pourrait être alors celui du texte; le déplacement, celui d'un regard mobile et réflexif sur les choses; l'altérité, celle du lecteur; l'expérience, celle liée au voyage dans/par l'écriture et la lecture: en somme, une expérience de l'altérité qui se construit aussi dans un rapport entre l'auteur et le lecteur, chacun incarnant des manières différentes d'être au monde et de l'expliquer. Je souhaiterais par là suggérer que, de l'articulation de ces deux formes de rapport à l'altérité altérité de l'objet d'étude, altérité du destinataire (le lecteur), dépend aussi, et ce, au travers de médiations complexes, la formulation géographique et savante d'expériences liées au déplacement.

BIBLIOGRAPHIE 
AUTHIER-REVUZ, J. (1982) - Hétérogénéité montrée et hétérogénéité constitutive: éléments pour une approche de l'autre dans le discours. DRLAV, 26: 91-151.

AUTHIER-REVUZ, J.(1984) - Hétérogénéité(s) énonciative(s). Langages, 19 (73): 98-111.

BERDOULAY, V. (1988) - Des mots et des lieux: la dynamique du discours géographique. Ed. du CNRS, Paris.

BRUnET, R. (1990) - Géographie Universelle, Hachette, Paris.

DematteIS, G. (1993) - Géographie universelle et géographie dite régionale. Espace Géographique, 22 (3): 255-280.

DuBOIS, Ph. (1981) - Le voyage et le livre. In CH. JACOB et al. (ed.). Arts et légendes d'espace. Figures du voyage et rhétoriques du monde. ENS, Paris.

GOMEZ MENDOZA, J.; N. ORTEGA CANTERo (1989) - L'approche régionale aujourd'hui. Géopoint, 88: 95-97.

MONDADA, L. (1994) - Verbalisation de l'espace et fabrication du savoir. Approche linguistique de la construction des objets de discours. Thèse de doctorat, Université de Lausanne.

VIDAl DE LA Blache, P. (1903) - Tableau de la Géographie de la France; Histoire de France sous la direction d'Ernest Lavisse, t. I. Hachette, Paris. 